\section{PS-066 OPEN SURGERY REPAIR IN INGUINAL HERNIA IN CHILDREN. A CLINICAL-EVOLUTIVE ASSESSMENT OF 64 PATIENTS}

C Berghea Neamtu. Pediatric Surgery, Pediatric Clinic Hospital "Lucian Blaga" University, Sibiu, Romania

\subsection{6/archdischild-2014-307384.362}

Background Open surgery (OS) repair represents the standard treatment in inguinal hernia (IH) in children, in whole world and still very well credited even though modern surgery provides interventional alternatives.

Aim Clinical-evolutive assessment of IH repair by OS in 20112013 period.

Methods The evaluation criteria of retrospective study were: age, environment provenance (EP), diagnosis at discharge, length of stay (LoS), surgical procedure protocol, cost of hospitalisation $(\mathrm{CoH})$ and complications.

Results 64 cases were evaluated: 45 (70,31\%) younger than 7 years old, $33(51,56 \%)$ originated from rural environment, 44 right side IH (4 strangulated, 5 scrotal-inguinal, 1 descends into labia) and 20 left side IH (2 scrotal-inguinal, 2 descend into labia), $40(62,5 \%)$ with LoS shorter than 5 days. The average LoS was 4,171 days. The surgery protocols record OS in all cases, 2 cases also requiring hematoma evacuation. Were recorded 8 postsurgical complications (5 hematoma, 3 scrotal oedema). Average $\mathrm{CoH}$ per day was $326,70 \mathrm{RON}$ and average $\mathrm{CoH}$ per patient $1324,63 \mathrm{RON}$.

IH were more frequent in patient younger than 7 years old from urban environment (p 0,08). Postsurgical complications were significantly more frequent in patient younger than 7 years old ( $\mathrm{p} 0,05$ ) without being influenced by IH side. There were no significant correlation between age and type of complication. Average $\mathrm{CoH}$ per day was negative influenced by complications (p 0,02) and positive by average LoS (p 0,09).

Conclusions The OS repair in IH in children still remains a frequently used procedure, implies fewer complications and not expensive costs.

\section{PS-067 TREATMENT OF VARICOCELE IN PAEDIATRIC PATIENTS WITH CONTRAINDICATION FOR OPEN SURGERY WITH TRANSFEMORAL RETROGRADE SCLERO-EMBOLIZATION UNDER LOCAL ANAESTHESIA}

${ }^{1} \mathrm{~N}$ Zampieri, ${ }^{2} \mathrm{RL}$ Castellani, ${ }^{2} \mathrm{G}$ Zampieri, ${ }^{1} \mathrm{FS}$ Camoglio. ${ }^{1}$ Department of Surgical Sciences Pediatric Surgical Unit University of Verona Policlinico G. B. Rossi Piazzale L. A. Scuro N.1 37134 Verona Italy, University of Verona, Verona, Italy; ${ }^{2}$ Department of Surgery, CASA Di Cura San Francesco, Verona, Italy

\subsection{6/archdischild-2014-307384.363}

Background Varicocele treatment in paediatric age is still under discussion, the aim of this study is to present our experience with transfemoral retrograde sclero-embolization in paediatric patients and varicocele under local anaesthesia with contraindication for open surgery.

Materials and methods Between December 2008 and December 2012184 patients 10 to 14 years of age with left varicocele were treated. Study inclusion criteria were created: grade II or III left varicocele; previous inguinal surgeries and contraindications to general anaesthesia. Sclero-embolization included the following procedures: right femoral access under local sedation (carbocaine). Mean time of intervention, recurrence and persistence rates as well as early and late complications were also considered.

Results During the study period 184 cases were treated with the radiological technique, 172 of which proved to be successful. In 10 cases a continent valve was found and it was therefore impossible to use this technique $(5,4 \%)$; Two cases had rupture of the vein with consequent spillage of the contrast agent. Twelve cases $(6,5 \%)$ showed recurrence No patient reported postoperative pain.

Discussion This technique proved to be efficient and reliable. It can be performed under local sedation and it involves fewer complications than traditional techniques.

The use of such technique allows preservation of the spermatic artery and is free from complications like testicular atrophy and hydrocele.

\section{PS-068 VARICOCELE AND ADOLESCENT: THE ROLE OF ELASTOSONOGRAPHY IN THE EVALUATION OF TESTES}

${ }^{1} \mathrm{~N}$ Zampieri, ${ }^{2} \mathrm{C}$ Bruno, ${ }^{1} \mathrm{~A}$ Mantovani, ${ }^{1} \mathrm{~S}$ Zambaldo, ${ }^{1} \mathrm{~A}$ Mariotto, ${ }^{1} \mathrm{M}$ Peretti, ${ }^{1} \mathrm{G}$ Scirè, ${ }^{1}$ FS Camoglio. 'Department of Surgical Sciences Pediatric Surgical Unit University of Verona Policlinico G. B. Rossi Piazzale L. A. Scuro N.1 37134 Verona Italy, University of Verona, Verona, Italy; ${ }^{2}$ Department of Radiology, University of Verona, Verona, Italy

\subsection{6/archdischild-2014-307384.364}

Background Varicocele is the first cause of male sub-fertility and it is well know its correlation with testicular growth arrest. In paediatric age testicular hypotrophy is the main indication for surgical treatment. The aim of this study is to evaluate the role of elastosonography in the evaluation of testicular elasticity as predictive sign of testicular damage.

Materials and methods 13 boys (9-13 years old) with untreated varicocele (bilateral in 3 boys and left unilateral in 10) and 12 age-matched healthy subjects underwent elastosonography. Varicocele was classified following the Dubin and Amelar classification and spermatic vein reflux was classified following a modified Hirsch classification (as short, medium and continuous spermatic vein reflux). The testicular elasticity was expressed as a three-point scale (1: normal; 2: slightly to moderately stiffer than normal; 3: severely stiffer than normal). None had testicular hypotrophy. Statistical analysis was performed by means of the Student's $t$-test.

Results 2 patients had grade I varicocele, II in 9 patients had grade II varicocele and 5 patients had grade III varicocele; 3 cases had "short $£$ spermatic vein reflux, 6 patients had "medium" spermatic vein reflux and 7 patients had continuous spermatic vein reflux. The elasticity was 1 in all 34 normal testes; in the testes with varicocele it was graded 2 in 9 cases and 3 in 7 cases. The differences in the degree of elasticity between normal testes and testes with varicocele and between continuous and medium/short spermatic vein reflux were highly significant ( $p<0.001$ in both cases); the difference between stage III and stages $\mathrm{I} / \mathrm{II}$ varicocele was just below significance ( $p$ $=0.053)$.

Conclusion Testes with varicocele are significantly stiffer than normal ones, with a positive correlation to the clinical grade and significantly to the duration of spermatic vein reflux. 


\section{PS-069}

\section{IN-HOSPITAL OUTCOMES FOLLOWING TRACHEOSTOMY IN INFANTS}

${ }^{1} \mathrm{HH}$ Lee, ${ }^{2} \mathrm{~PB}$ Smith, ${ }^{3} \mathrm{BH}$ Quek, ${ }^{4} \mathrm{RH}$ Clark, ${ }^{5} \mathrm{CP}$ Hornik. ${ }^{1}$ Children's Intensive Care Unit, KK Women's and Children's Hospital, Singapore, Singapore; ${ }^{2}$ Neonatal-Perinatal Medicine, Duke Children's Hospital, Durham, USA; ${ }^{3}$ Neonatology, KK Women's and Children's Hospital, Singapore, Singapore; "Neonatal Medicine, Pediatrics Medical Group, Greenville, USA; ${ }^{5}$ Pediatric Critical Care Medicine, Duke Children's Hospital, Durham, USA

\subsection{6/archdischild-2014-307384.365}

Background and aims Tracheostomy is performed in infants with airway anomalies or requiring prolonged mechanical ventilation (MV). Risks and outcomes are described only in small studies. We report risk factors for mortality following tracheostomy in a large cohort of infants.

Methods We identified all infants discharged from 348 NICUs managed by Pediatrix Medical Group who underwent tracheostomy between 1997 and 2012. We only included infants cared for at a single site. We performed multivariable logistic regression with random effects for site to evaluate association between death after tracheostomy and risk factors: diagnosis, gestational age, small for gestational age (SGA), age at tracheostomy, and days exposed to fraction of inspired oxygen $>40 \%$, inotropes and MV prior to tracheostomy.

Results 532 infants required tracheostomy $(0.06 \%$ of infants). Median gestational age and birth weight were 26 weeks (IQR; $25,30)$ and $780 \mathrm{~g}(610,1400)$. The most common diagnoses were bronchopulmonary dysplasia, 465/532 (85\%), airway anomalies, $237 / 532(45 \%)$ and pulmonary anomalies, 88/532 (17\%). Tracheostomy was performed on median postnatal age of 87 days $(36,128)$. Of the 532 infants, 344 (65\%) were weaned off MV prior to discharge at a median of 6 days $(3,12)$ after tracheostomy. Mortality was $14 \%$. On multivariable regression, the following were associated with mortality: days of oxygen exposure, $\mathrm{OR}=1.01$ (95\% CI; 1.00, 1.02); inotrope exposure, $\mathrm{OR}=1.04(1.00,1.09) ;$ SGA, OR $=2.40(1.32,4.35)$.

Conclusion While tracheostomy is rarely performed, mortality after the procedure is high and is associated with increased by pre-tracheostomy oxygen and inotrope exposures and SGA status.

\section{PS-070 FUNCTIONAL ENDOSCOPIC SINUS SURGERY IN CHILDREN}

M Maniuc, P Ababii, D Chirtoca, S Diacova, L Danilov. Otorhinolaryngology, State Medical and Pharmaceutical University Nicolae Testemitanu, Chisinau, Moldova

\subsection{6/archdischild-2014-307384.366}

The aim of the study was the optimisation of surgical treatment in children with chronic and recurrent [em1] rhinosinusitis. The work is based on comparative study of results after surgical treatment of 289 children with recurrent and chronic rhinosinusitis. The age of the children ranged between 3-15 years old, 188 males (65\%) and 101 females (35\%).

Methods Inconformity with the method of the treatment all the patients were divided in 2 groups. In the patients of the first group (84 children) the standard endoscopic sinus surgery was performed. This method includes: the relatively large ablation of all the anatomical structures from operation zone (processus uncinatus, ethmoid cells, ostium of sinus maxillary). In the patients of the second group (205 children) the minimally invasive endoscopic sinus surgery was performed. This modification includes partial resection of processus uncinatus and limited ablation of all the anatomical structures of ostiomeatal complex. Results The duration of the treatment in patients from the I group was 6,15 $\pm 0,24$ days, in the children from the II group it was4, $26 \pm 0,25$ days, with statistic differences between the II and the I group $(t=7,15 ; \mathrm{p}<0,001)$. The patients were considered recovered if all the symptoms were absent over a period of 36 months. The good results were determined in $73,7 \%$ of the patients in group I and in $85,7 \%$ in the group II.

Conclusion The best results were obtained in patients who have undergone the minimally invasive endoscopic sinus surgery.

\section{PS-071 MODIFIED SURGERY IN CHILDREN WITH PERSISTENT AND RECURRENT OTITIS MEDIA}

'S Diacova, ${ }^{1}$ I Ababii, 'M Maniuc, 'L Danilov, ${ }^{1} \mathrm{P}$ Ababii, ${ }^{2} \mathrm{O}$ Diacova, ${ }^{3} \mathrm{TJ}$ McDonald. ${ }^{1}$ Otorhinolaryngology, State University of Medicine and Pharmacy "N. Testemitanu", Chisinau, Moldova; ${ }^{2}$ Student, State University of Medicine and Pharmacy "N. Testemitanu", Chisinau, Moldova; ${ }^{3}$ Otorhinolaryngology, Mayo Clinic, Rochester, USA

\subsection{6/archdischild-2014-307384.367}

Background and aims The effectiveness of classical tympanostomy in persistent and recurrent otitis media (OM) with presence of mucous effusion during surgery is unclear. We elaborated the modified technique of surgery in order to improve the complete aspiration of viscous content from posterior part of tympanic cavity. This study was conducted to determine the effectiveness of the modified type of tympanostomy. Methods A total of 67 children (134 ears) with OM and mucous viscous content during the myringotomy were included in Project. All cases were divided into two groups according to technique: simple (Group S) versus modified (Group M). Clinical and audio logical examination was performed every 3 months during 1 year after surgery. Otomicroscopical evaluation of ears was done under general anaesthesia in 12 months after tympanostomy, at the time of tube removal. Presence of retractions, adhesions, granulation tissue and effusion was noted.

Results Recurrence of OM was characteristic for $19 \%$ of ears from Group S and 2\% of ears from Group M. Undulating hearing loss was recorded in Group S in 34\% of cases, comparing to $7 \%$ of Group M. Attic retraction and adhesions in Group S were noted in 14\%, in Group M - in 3\% of cases.

Conclusion Modified technique of tympanostomy is more effective than simple one in preventing of hearing loss, formation of attic retraction, adhesions, recurrence of effusion and granulation tissue in children with persistent and recurrent $\mathrm{OM}$ and presence of mucous effusion during surgery.

\section{PS-072 ASSESSMENT OF FACTORS AFFECTING PRE-OPERATIVE ANXIETY AND COMPLIANCE TO ANAESTHESIA INDUCTION IN SCHOOL-GOING CHILDREN}

${ }^{1} \mathrm{P}$ Mathew, ${ }^{1} \mathrm{RH}$ Malik, ${ }^{1} \mathrm{~S}$ Yaddanappudi, ${ }^{2} \mathrm{~A}$ Kohli, ${ }^{1} \mathrm{NB}$ Panda. ${ }^{1}$ Anaesthesia and Intensive Care, Postgraduate Institute of Medical Education and Research, Chandigarh, India; ${ }^{2}$ Psychiatry and Clinical Psychology, Postgraduate Institute of Medical Education and Research, Chandigarh, India

\subsection{6/archdischild-2014-307384.368}

Background and aims Effective pre-operative preparation program to address child's anxiety and thus improve the quality of anaesthetic induction would need to identify the factors that affect pre-op anxiety. The factors that influence pre-operative 